\section{Fluorescence Produced by Transfection Reagents Can Be Confused with Green Fluorescent Pro- teins in Mammalian Cells}

\author{
BioTechniques 31:314-321 (August 2001)
}

\section{ABSTRACT}

The Aequorea victoria green fluorescent protein (GFP) reporter system is a convenient way to monitor gene expression and other cellular functions in mammalian cells. To study gene expression, a GFP-fusion plasmid construct is often transfected into mammalian cells using a variety of methods including calcium phosphate- and liposome-based DNA transfer. Subsequent$l y$, the expression of GFP-fusion protein is monitored by fluorescence microscopy or flow cytometry. Here, we report that certain transfection reagents can produce fluorescence that can be detected in a wide range of wavelengths, which can be confused with GFP-fusion protein. The fluorescence false positives can be a problem, particularly when the GFP expression levels are low. To improve the GFP-based detection or screening methods, it is imperative to include an appropriate negative control and to detect GFP using a narrow-wavelength emission filter corresponding to the emission spectrum around the GFP peak.

\section{INTRODUCTION}

Transient transfection is often used to study gene expression or promoter functions in mammalian cells into which plasmid constructs have to be delivered. A wide variety of techniques and reagents have been developed to effectively deliver DNA into mammalian cells. Among these, calcium phosphate co-precipitation $(5,12,18)$ and liposomebased DNA delivery systems $(2,9,10,17)$ are most widely used because they are easy to use and can achieve relatively high transfection efficiencies.

A variety of reporter systems have been developed to monitor the expression and biological functions of genes, which includes chloramphenicol acetyltransferase (11), $\beta$-galactosidase (13), luciferase (7), and Aequorea victoria green fluorescent protein (GFP) (4). Among these, the GFP reporter system has many advantages. Perhaps the most important advantage is that the GFP tag allows the monitoring of gene expression within living cells. It is even possible to monitor the location of the GFP-fusion proteins in the cytoplasm, nucleus, or other subcelluar compartments such as the mitochondria of the living cells (16). Furthermore, the interactions of two or more different proteins can be monitored in the living cells using fusion proteins tagged with different GFP mutants that can produce different fluorescent signals $(8,14)$.

Recently, high-throughput screening methods using a GFP tag were used in drug discovery and other industrial applications $(1,3,15,19)$. A high-throughput approach is very efficient in screening a large number of samples in a given time. However, this type of GFPbased screening method can be a problem when the GFP expression levels are low. The problem can be com pounded when the transfection reagents show substantial fluorescence by them selves or by interacting with cellular components. Although this problem can be potentially serious, little data have been reported on this subject. To gain a better understanding about whether transfection reagents can affect the interpretation of experimental results, we have systematically investigated the effects of three commonly used transfection reagents, calcium phosphate and two commercially available liposome-based transfection reagents (reagents $\mathrm{A}$ and $\mathrm{B}$, respective1y). We have found that the fluorescence produced by these reagents can be seriously misleading in the interpretation of the experimental data under certain circumstances.

\section{MATERIALS AND METHODS}

\section{Cells, Plasmid, and Transfection}

Chinese hamster ovary (CHO) cells were maintained in MEM supplemented with $10 \%$ Fetal Clone ${ }^{\circledR}$ II serum (HyClone Laboratories, Logan, UT,
USA). MDA-MB231 human breast cancer cells were maintained in RPMI 1640 containing 10\% FBS (HyClone Laboratories). The plasmid pEGFP-N1 (Clontech Laboratories, Palo Alto, CA, USA) was used in transfection assays as the positive control.

To prepare the samples for transfection, the cells were grown in a $10-\mathrm{cm}$ dish containing two or three sterile glass coverslips. Each of three sets consisted of six dishes. One of the six dishes was transfected with pEGFP-N1 using calcium phosphate co-precipitation or reagents A or B (positive control). For a negative control, both the plasmid and transfection reagent were substituted with the same volumes of sterile distilled water. The other four were mock transfected (i.e., no plasmid included) using one of the three transfection reagents. Transfection was done when the cells reached approximately $30 \%$ confluence.

The transfection assays followed the protocols provided by the manufacturers with some modifications. Briefly, for transfection with cationic lipid reagents, $30 \mu \mathrm{L}$ reagents $\mathrm{A}$ or $\mathrm{B}$ were mixed with $120 \mu \mathrm{L}$ water in a microtube. Two micrograms of plasmid DNA diluted in $150 \mu \mathrm{L}$ water were prepared in a separate microtube. The two solutions were then mixed together and incubated at room temperature for 15-20 min (i.e., liposome-DNA mixture). The cells to be transfected were washed twice with pre-warmed, serum-free medium, and then $4.5 \mathrm{~mL}$ serum-free medium were added to each $10-\mathrm{cm}$ culture dish. The liposome-DNA mixture (or liposome-water for mock transfection) was added to the culture medium in a dropwise fashion. After $5 \mathrm{~h}$ of incubation at $37^{\circ} \mathrm{C}, 5 \mathrm{~mL}$ medium containing $20 \%$ serum were added to the culture dish to achieve the final serum concentration of $10 \%$. The medium was replaced with fresh complete medium the following day. For the calcium phosphate-mediated transfection, $5 \mu \mathrm{g}$ plasmid DNA (or the same volume of water for mock transfection) in $438 \mu \mathrm{L}$ water were mixed with $61 \mu \mathrm{L}$ $2.0 \mathrm{M} \mathrm{CaCl} 2$. To this, $500 \mu \mathrm{L} 2 \times$ HEPES buffer (274 mM NaCl, $1.5 \mathrm{mM}$ $\mathrm{Na}_{2} \mathrm{HPO}_{4}$, and $54.5 \mathrm{mM}$ HEPES, $\mathrm{pH}$ 7.0) were slowly mixed. The resulting mixture was then added to the cell cul- 


\section{Short Technical Reports}

ture in a dropwise fashion, after which the cells were identically treated as in the liposome-based transfection.

\section{Fluorescence Microscopy}

Twenty-four hours after transfection, the cells on the glass coverslips were washed twice with PBS $(2.7 \mathrm{mM}$ $\mathrm{KCl}, 1.0 \mathrm{mM} \mathrm{KH}_{2} \mathrm{P} \mathrm{O}_{4}, 137 \mathrm{mM} \mathrm{NaCl}$, and $10 \mathrm{mM} \mathrm{Na} 2 \mathrm{HPO}_{4}, \mathrm{pH} \mathrm{7.4)} \mathrm{and}$ were then examined using a Zeiss ${ }^{\circledR}$ Axiovert 100 inverted microscope (Carl Zeiss, Thornwood, NY, USA). The filters used in the experiments were Cyan GFP (exciter D436/20x and emitter D480/40m), CFP/YFP (exciter 51017x and emitter $51017 \mathrm{~m}$ ), Endow GFP Bandpass (exciter HQ470/40x and emitter HQ525/50m), Rhodamine Red $^{\mathrm{TM}}$ (exciter D540/25x and emitter D605/55m), and $\mathrm{Cy}^{\mathrm{TM}}$ (exciter HQ620/60x and emitter HQ700/75m) (all filters are from Chroma Technology, Brattleboro, VT, USA). For each sample, approximately 50 cells were examined to estimate the transfection efficiency. Images of the cells were taken consecutively with several filters at $63 \times$ magnification using an Optronics 3 CCD cooled color camera (Carl Zeiss). The image data were processed using the Northern Eclipse software (Empix Imaging, Mississauga, ON, Canada).

\section{Flow Cytometric Analysis}

To prepare the cells for flow cytometric analysis, the cells were trypsinized and suspended in $1 \times$ PBS. For each sample, approximately $3 \times 10^{4}$ cells were analyzed using an Epics ${ }^{\circledR}$ Elite flow cytometer (Beckman Coulter, Fullerton, CA, USA). Fluorescence intensity upon stimulation with an argon-ion laser at $488 \mathrm{~nm}$ was measured between 500 and $540 \mathrm{~nm}$ and plotted against the cell number. The fluorescence emission of untransfected, mocktransfected, and plasmid-transfected cells were compared using Multiplus AV software (Phoenix Flow Systems, San Diego, CA, USA). The relative fluorescence intensity was determined by subtracting the signal of untransfected cells from the signal of the transfected or mock-transfected cells.

\section{RESULTS}

As expected, neither $\mathrm{CHO}$ nor MDA-MB231 cells showed significant cellular autofluorescence with any of the filters when the cells were mock transfected with water (Figure 1, rows I and i). Thus, cellular autofluorescence was not a problem under our experimental conditions. The GFP expressed
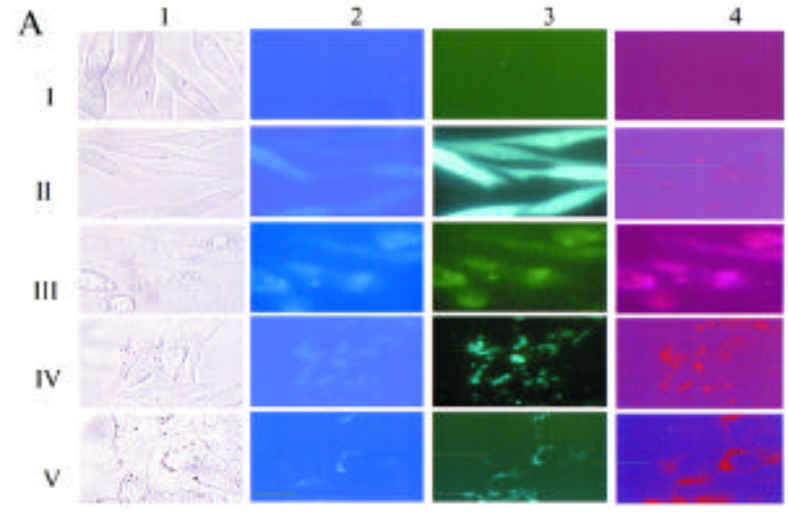

B

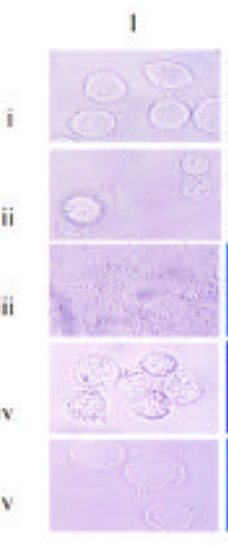

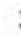
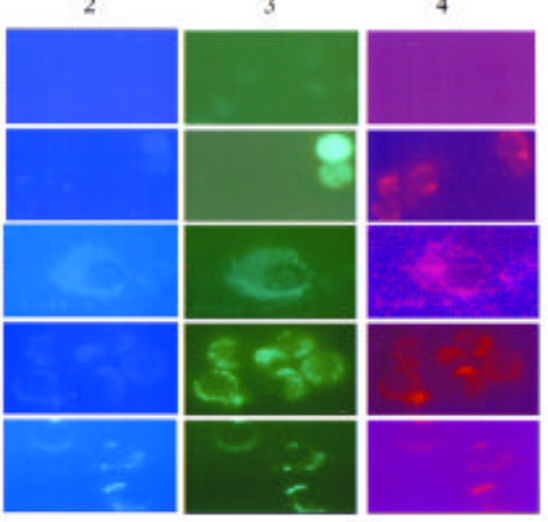
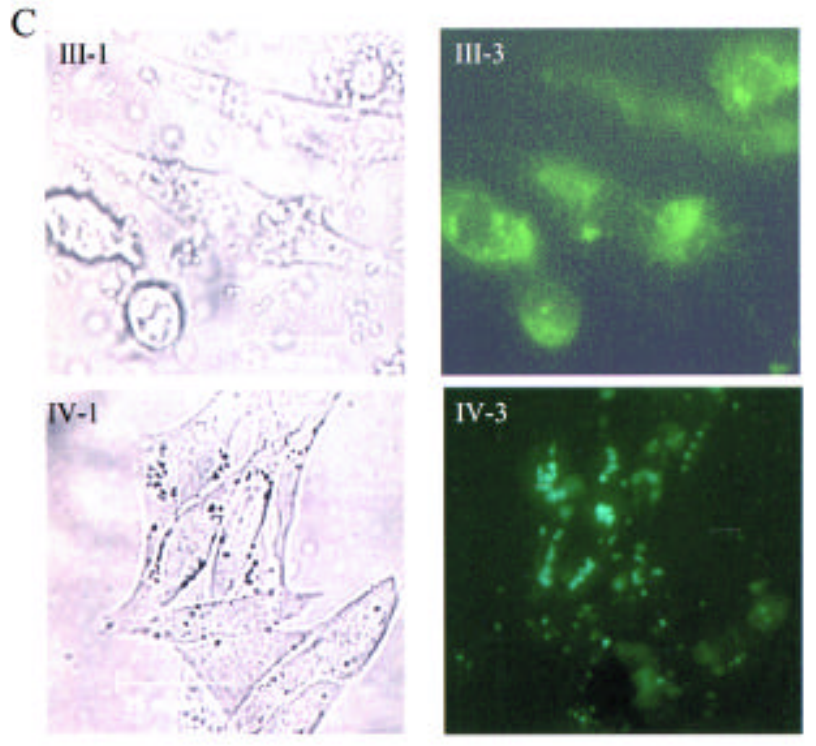

Figure 1. Transfection reagents produce fluorescence in a wide range of spectra. $\mathrm{CHO}$ (A) and MDA-MB231 (B) cells were transfected as follows: rows I and i, mock-transfected samples using water only; rows II and ii, transfected with pEGFP-N1 using reagent A; rows III, iii, IV, iv, V, and v, mock-transfected samples using calcium phosphate, reagents A or B, respectively. For photography, the following filters were used: column 1, field light; column 2, Chroma Cyan GFP; column 3, Chroma Endow GFP Bandpass; and column 4, Chroma Cy5. To show more details of Figure 1, panels III-1, III-3, IV-1, and IV-3, are enlarged in Figure 1C. 
by pEGFP-N1 (positive control) in both CHO and MDA-MB231 cells showed a typical signal that was evenly distributed within the whole cells when any one of the transfection reagents was used and detected through the Endow GFP Bandpass filter (Figure 1, panels II-3 and ii-3). The positive control also showed fluorescent signals with the Cyan GFP filter, although they were weak (Figure 1, panels II-2 and ii-2). It was also noted that the positive control showed distinct fluorescent signals with the Cy5 filter (Figure 1, panels II4 and ii-4). However, the fluorescence was not from the GFP but from the transfection reagent, because fluorescence was detected in the cytoplasm of both transfected (Figure 1B, one cell at the top right in panel ii-4) and untransfected cells (Figure 1B, the three cells at left in panel ii-4).

Calcium phosphate precipitation alone generated fluorescence when the mock transfection was performed (Figure 1, rows III and iii). This fluorescent signal had a much lower intensity than the GFP signal; nevertheless, it was easily detected by fluorescence microscopy. The fluorescence generated by calcium phosphate looked like patches on the cell surface. These fluorescent patterns are in stark contrast with those generated by the liposome-based transfection reagents A or B (Figure 1, rows IV, V, iv, and v, and Figure 1C, panels III-3 and IV-3). Cells with large patches of calcium phosphate precipitation usually died within 2-3 days. The cells with small patches of precipitate showed fluorescent signals in granular shapes or irregular patches and usually

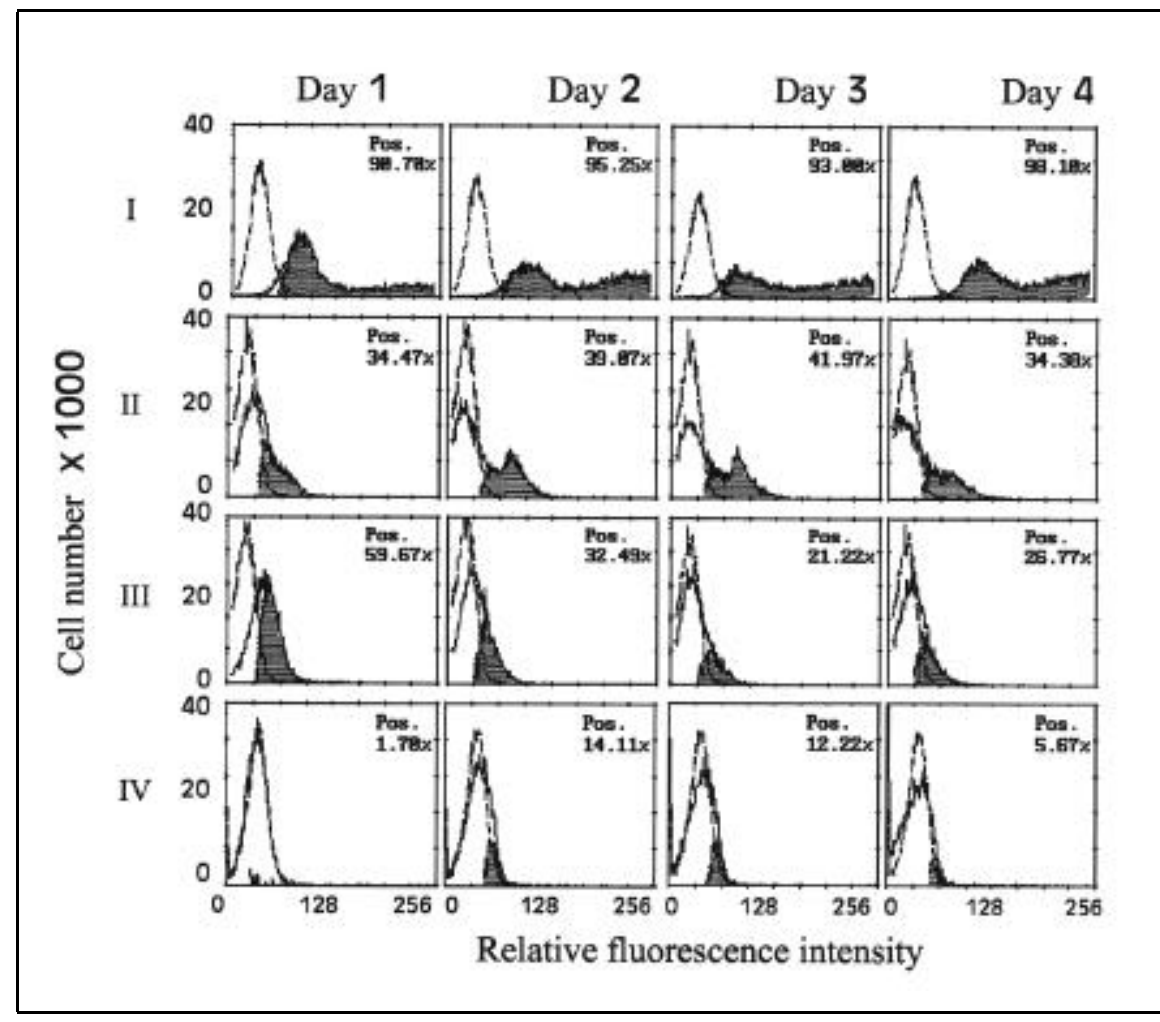

Figure 2. Fluorescence emitted by transfection reagents can be confused with GFP expression in the flow cytometric analysis. The negative controls (open peak shown at left in each panel) represent the cells that were mock transfected with water (i.e., rows I-IV). Each row represents data generated from CHO cells using the following transfection methods: row I (positive control), cells transfected with pEGFP-N1 using reagent A; rows II, III, and IV, mock-transfected samples using calcium phosphate reagents A or B, respectively. The columns (Days) represent sampling time after mock transfection. The numbers in the panels are the number of positively identified cells expressed in percentages. The numbers in the $\mathrm{X}$ axis represent arbitrary channel numbers. The original acquisition of the fluorescence intensity of each cell was in a log scale. However, the cells with different fluorescence intensities were arbitrarily assigned into 256 channels in a linear fashion. Therefore, the cells in the channel with higher numbers indicate that they emit higher fluorescence signals. However, the numbers themselves do not represent the original fluorescence intensity of each cell in a linear or a log scale but relative fluorescence intensity.

survived the transfection procedure.

Over $80 \%$ of the cells that were mock transfected with the liposomebased transfection reagents showed fluorescent signals in their cytoplasm, although the intensity of the signals in many of these cells was too low to be detected by the flow cytometric analysis (Figure 2). The liposome-mediated autofluorescence usually appeared as small freckles or granules around the nucleus, as shown in Figure 1A, rows IV and V, Figure 1B, rows $v$ and vi, and Figure 1C, panel IV-3, respectively. The number of granules and the intensity of fluorescence generated by the reagent $\mathrm{A}$ were much more readily detected under the fluorescence microscope. Conversely, the signals produced by the reagent $\mathrm{B}$ were low, both in the number of granules and in the intensity of the fluorescence. Interestingly, the fluorescent granules generated by the liposome-based reagents often coincided with the dark spots observed under a bright field without any filter (Figure 1, A and B, panels IV-1, V-1, iv-1, and $\mathrm{v}-1$, respectively). This may suggest that the fluorescence is from the liposome-based transfection reagents trapped in certain subcellular compartments such as cytoplasmic endosomes or lysosomes (6).

Fluorescence was detected in both the $\mathrm{CHO}$ and MDA-MB231 cells that had been mock transfected using calcium phosphate or reagents A or B (Figure 1). In all of these cases, fluorescence was stable up to four days and detected under all filter sets used. The exposure time with the "blue" and "green" filters was $0.25-0.5 \mathrm{~s}$. However, the Cy5 filter usually took a much longer exposure time (4-8 s) to detect the same level of fluorescent images. The data generated using yellow and Rhodamine Red filters were very similar to those shown in Figure 1 (data not shown).

To determine the overall effects of the transfection reagents on the detection of GFP expression in the entire cell population, we carried out flow cytometric analysis after $\mathrm{CHO}$ cells were mock transfected. Consistent with the analysis done by fluorescence microscopy on individual cells, a substantial number of cells among the mock-transfected population using calcium phosphate or reagent A showed fluorescence 
$24 \mathrm{~h}$ after the mock transfection (Figure 2, panels II-Day 1 and III-Day 1).

The delivery of pEGFP-N1 into the $\mathrm{CHO}$ cells by reagent A seemed very effective because the positive rates were over $90 \%$ (Figure 2, row I). How ever, these numbers can be misleading because the positive fluorescent signal from the cells that were mock transfected with reagent A was substantial (Figure 2, row III). The number of false positives in the mock-transfected cells using reagent $A$ had decreased by half by Day 2 . However, the levels of the fluorescence were still 20\%-30\% up to Day 4 (Figure 2, row III). Interestingly, the relative number of false positives did not substantially decrease in the mock-transfected cells using calcium phosphate up to Day 4 after transfection (Figure 2, row II). In contrast to the calcium phosphate- and the reagent Amediated transfection, reagent $\mathrm{B}$ showed much lower false-positive rates when analyzed by flow cytometry (Figure 2, row IV). However, the overall transfection efficiency of this reagent was usually less than $20 \%$ for both CHO and MDA-MB231 (data not shown) compared to $40 \%-70 \%$ transfection efficiency produced by the reagent A-based transfection.

\section{DISCUSSION}

The GFP reporter system has become a powerful tool in studying protein and promoter functions in living cells and is often delivered into mam malian cells using calcium phosphateor liposome-based reagents. Furthermore, the GFP-based detection method is increasingly used for a high-throughput screening in drug discovery and other industrial applications. Unfortunately, we found that these transfection reagents can produce substantial fluorescent signals, which can mislead the interpretation of experimental data generated by a GFP reporter system. For example, transfection carried out by either calcium phosphate co-precipitation or reagent A can artificially exaggerate transfection efficiency because of the false-positive signals detected by flow cytometry (Figure 2). Under our experimental conditions, the calcium phosphate co-precipitation method caused the most serious problems in both the false-positive rates and fluorescence intensity (Figure 1). The interpretation of transfection efficiency can be a more serious problem when the GFP expression levels are low. Reagent $B$ is superior in this aspect because its false-positive signals were much lower (Figure 2, row IV). However, the overall transfection efficiency of this reagent was generally low under our experimental conditions.

In addition, confusion can arise when a GFP-fusion protein is used to study its subcellular localization and interactions with other proteins. Liposome-based transfection may be particularly problematic in this aspect because both reagents $\mathrm{A}$ and $\mathrm{B}$ can generate pronounced fluorescent signals in the discrete subcellular locations (Figure 1). The problem can be further complicated because the discrete signals generated by these reagents are observed in a wide range of wavelengths (filters).

It is interesting to note that reagent A alone does not show fluorescence. Therefore, this reagent may interact with certain cellular components to produce fluorescence. If this is the case, then the cellular components that interact with reagent $\mathrm{A}$ would be abundant only in certain parts of subcellular locations, as shown in Figure 1, A, B, and $C$, rows IV and iv, respectively, and panel IV-3. This possibility can further complicate the interpretation of data with respect to protein localization, protein-protein interactions, and other protein functions. Therefore, the identification of the components that can interact with transfection reagents and thus generate fluorescence may be necessary to further refine the liposomebased transfection method.

It is important to note that the transfection reagent-mediated fluorescence was detected over a wide range of wavelengths (Figure 1). The problem caused by false positives can be particularly serious when the wavelengths of the emission filters used for the fluorescence detection are either much shorter or much longer than the emission peaks of the GFP used. For example, when the Chroma Cyan GFP filter (exciter D436/20x and emitter D480/40m) was used to detect the enhanced GFP (EGFP) fluorescence (peak at $507 \mathrm{~nm}$ ), false positives often emitted stronger signals than the positive cells (Figure 1, panel III-2 versus panel II-2). The same problem exists when a long wavelength filter (e.g., Chroma Cy5; exciter HQ620/60x and emitter HQ700/75m) was used to detect the EGFP fluorescence (Figure 1, panel III-4 versus panel II-4). Therefore, using narrow emission filters corresponding to the emission spectra around the GFP peaks (507 $\mathrm{nm}$ for EGFP, $433 \mathrm{~nm}$ for ECFP, and $527 \mathrm{~nm}$ for EYFP) can significantly reduce false positives.

When GFP expression is analyzed by flow cytometry, it is important to include mock-transfected control using the same transfection reagent. The portion of false positive signals should be taken into account in the final flow cytometry data. Furthermore, when developing a high-throughput protocol, it will be imperative to thoroughly investigate the false-positive signals caused by transfection reagents under experimental conditions using both fluorescence microscopy and flow cytometry before performing a large-scale screen.

\section{REFERENCES}

1.Berrie, C.P. and M. Falasca. 2000. Patterns within protein/polyphosphoinositide interactions provide specific targets for therapeutic intervention. FASEB J. 14:2618-2622.

2. Bertling, W.M., M. Gareis, V. Paspaleeva, A. Zimmer, J. Kreuter, E. Nurnberg, and P. Harrer. 1991. Use of liposomes, viral capsids, and nanoparticles as DNA carriers. Biotechnol. Appl. Biochem. 13:390-405.

3.Brock, R., G. Vamosi, G. Vereb, and T.M. Jovin. 1999. Rapid characterization of green fluorescent protein fusion proteins on the molecular and cellular level by fluorescence correlation microscopy. Proc. Natl. Acad. Sci. USA 96:10123-10128.

4.Chalfie, M., Y. Tu, G. Euskirchen, W.W. Ward, and D.C. Prasher. 1994. Green fluorescent protein as a marker for gene expression. Science 263:802-805.

5.Chen, C. and H. Okayama. 1987. High-efficiency transformation of mammalian cells by plasmid DNA. Mol. Cell. Biol. 7:2745-2752.

6.Coonrod, A., F.Q. Li, and M. Horwitz. 1997. On the mechanism of DNA transfection: efficient gene transfer without viruses. Gene Ther. 4:1313-1321.

7.de Wet, J.R., K.V. Wood, M. DeLuca, D.R. Helinski, and S. Subramani. 1987. Firefly luciferase gene: structure and expression in mammalian cells. Mol. Cell. Biol. 7:725-737.

8.Ellenberg, J., J. Lippincott-Schwartz, and J.F. Presley. 1999. Dual-colour imaging with GFP variants. Trends Cell Biol. 9:52-56.

9.Felgner, P.L., T.R. Gadek, M. Holm, R. Ro- 
man, H.W. Chan, M. Wenz, J.P. Northrop, G.M. Ringold et al. 1987. Lipofection: a highly efficient, lipid-mediated DNA-transfection procedure. Proc. Natl. Acad. Sci. USA 84: 7413-7417.

10.Gershon, H., R. Ghirlando, S.B. Guttman, and A. Minsky. 1993. Mode of formation and structural features of DNA-cationic liposome complexes used for transfection. Biochem. 32:7143-7151.

11.Gorman, C.M., L.F. Moffat, and B.H. How ard. 1982. Recombinant genomes which express chloramphenicol acetyltransferase in mammalian cells. Mol. Cell. Biol. 2:1044-1051.

12.Graham, F.L. and A.J. van der Eb. 1973. A new technique for the assay of infectivity of human adenovirus 5 DNA. Virology 52:456467.

13.Hall, C.V., P.E. Jacob, G.M. Ringold, and F. Lee. 1983. Expression and regulation of Escherichia coli lacZ gene fusions in mammalian cells. J. Mol. Appl. Genet. 2:101-109.

14.Heim, R., D.C. Prasher, and R.Y. Tsien. 1994. Wavelength mutations and posttranslational autoxidation of green fluorescent protein. Proc. Natl. Acad. Sci. USA 91:1250112504.

15.Motoike, T., S. Loughna, E. Perens, B.L. Roman, W. Liao, T.C. Chau, C.D. Richardson, T. Kawate et al. 2000. Universal GFP reporter for the study of vascular development. Genesis 28:75-81.

16. Rizzuto, R., M. Brini, F. De Giorgi, R. Rossi, R. Heim, R.Y. Tsien, and T. Pozzan. 1996 Double labelling of subcellular structures with organelle-targeted GFP mutants in vivo. Curr. Biol. 6:183-188.

17.Smith, J.G., R.L. Walzem, and J.B. German. 1993. Liposomes as agents of DNA transfer. Biochim. Biophys. Acta 1154:327340.

18.Wigler, M., A. Pellicer, S. Silverstein, R. Axel, G. Urlaub, and L. Chasin. 1979. DNAmediated transfer of the adenine phosphoribosyltransferase locus into mammalian cells. Proc. Natl. Acad. Sci. USA 76:1373-1376.

19.Zhu, M. and W.E. Fahl. 2000. Development of a green fluorescent protein microplate assay for the screening of chemopreventive agents. Anal. Biochem. 287:210-217.

We thank the members of the Lee laboratory for their many helpful discussions during our laboratory meetings. This work was supported by grants from the MRC-C/CIHR (no. MT-15015) and the Northern Ontario Heritage Fund (no. S7AHL59) to H.L. and by a grant from the Northern Cancer Re- search Foundation (no. S7AHL096) to B.G. Address correspondence to Dr. Hoyun Lee, Department of Research, Northeastern Ontario Regional Cancer Centre, 41 Ramsey Lake Road, Sudbury, ON, Canada P3E 5J1. e-mail: hlee@neorcc.on.ca

Received 1 September 2000; accepted 23 February 2001.

\section{B. Guo, A.G. Pearce, K.E.A.} Traulsen, A.C. Rintala, and H. Lee Northeastern Ontario Regional Cancer Centre Sudbury, ON, Canada

For reprints of this or any other article, contact Reprints@BioTechniques.com 